Diagnostic challenges in a case of B-cell lymphoma unclassifiable with features intermediate between diffuse large B-cell lymphoma and Burkitt lymphoma

\title{
Chi-Chiu So*
}

Department of Pathology, Faculty of Medicine, The University of Hong Kong, Hong Kong SAR, China

\section{Ka-Hung Yung}

Department of Hematology, Centro Hospitalar Conde de Sao Januario, Macau SAR, China

\section{Man-Leng Chu}

Department of Anatomic Pathology, Centro Hospitalar Conde de Sao Januario, Macau SAR, China

Thomas SK Wan

Department of Pathology, Faculty of Medicine, The University of Hong Kong, Hong Kong

*Corresponding author: Dr. Chi-Chiu So, Department of Pathology, Faculty of Medicine, The University of Hong Kong. Email: scc@pathology.hku.hk; tel: 852-22554570; fax: 852-28177565.

Running title: Genetic diagnosis in lymphoma

Key words: B-cell lymphoma, cytogenetics

Word count: 1217

Number of tables: 0

Number of figures: 2 composite 
Abstract Unclassifiable B-cell lymphoma with features intermediate between diffuse large B-cell lymphoma (DLBCL) and Burkitt lymphoma (BL) is a recently recognized category of mature B-cell lymphoma. This represents a heterogeneous group of diseases which often pose diagnostic problems in clinical practice, yet its distinction from DLBCL and BL is important for its therapeutic and prognostic implications. We report the challenging diagnostic process of a 60 -year old man. He had a CD10 and BCL2-positive, BCL6-negative B-cell malignancy with loss of multiple B-cell markers including surface immunoglobulin. The karyotype was complex and unusual, including $\mathrm{t}(2 ; 18)(\mathrm{p} 12 ; \mathrm{q} 21), \mathrm{t}(8 ; 14)(\mathrm{q} 24 ; \mathrm{q} 32)$ and a derivative chromosome 22 mimicking a Philadelphia chromosome that led to initial diagnostic confusion. A triple-hit gray zone B-cell lymphoma with rearrangements of $M Y C$, BCL2, BCL6, both alleles of IGH and likely IGK and IGL was finally diagnosed upon additional fluorescence in-situ hybridization (FISH) studies. His disease was nonresponsive to intensive combination chemotherapy and he died four months after presentation. This case illustrates the diagnostic difficulty encountered in such group of B-cell lymphomas and emphasizes the need to integrate morphological, immunophenotypic and genetic data in making a diagnosis. 


\section{Introduction}

B-cell lymphoma unclassifiable with features intermediate between DLBCL and BL is a newly introduced category of mature B-cell lymphoma in the 2008 updated WHO Classification [1]. It is a heterogeneous group of lymphomas found mostly in elderly patients with advanced stage disease [2]. Tumor morphology and/or immunophenotype are intermediate between BL and DLBCL. Diagnosis is often challenging due to lack of a specific morphologic, immunophenotypic and genetic pattern. Prognosis is very poor as they respond neither to BL- or DLBCL-type chemotherapeutic regimens [2]. Distinction from BL and DLBCL is therefore very important. We describe this diagnosis in an elderly man whose disease showed an unusual karyotype. 


\section{Case report}

The patient was a 60-year old man who complained of fever for three weeks. Physical examination detected splenomegaly. There was no hepatomegaly or peripheral lymphadenopathy. Screening blood investigations showed mild anemia (hemoglobin $103 \mathrm{~g} / \mathrm{L}$ ), moderate thrombocytopenia (platelet $74 \times 10^{9} / \mathrm{L}$ ), raised liver enzymes (alanine aminotransferase $100 \mathrm{U} / \mathrm{L}$, aspartate aminotransferase $186 \mathrm{U} / \mathrm{L}$ ) and markedly elevated lactate dehydrogenase (LDH) (7500 U/L). No abnormal white blood cells were seen on peripheral blood smear examination. Computed tomography confirmed splenomegaly. In addition, mediastinal lymphadenopathy was observed.

Bone marrow aspiration cytology showed many malignant cells that were heterogeneous in size, with a high nuclear-to-cytoplasmic ratio, slightly irregular nuclei, condensed chromatin, single to multiple small nucleoli and a rim of deep basophilic cytoplasm that occasionally contained clear vacuoles (Fig. 1a). The cytological finding of condensed chromatin in the malignant cells is more in favor of a high grade lymphoma than an acute leukemia. Presence of distinct cytoplasmic vacuoles within the intensely basophilic cytoplasm of the malignant cells raised the suspicion of a BL. Although the size heterogeneity and nuclear irregularity are atypical of BL, findings from gene expression profiling studies have demonstrated that lymphomas with a typical expression signature of BL can have a relatively wide 
morphological spectrum $[3,4]$.

Flow cytometric analysis revealed an abnormal population of B cells expressing CD10, CD19, CD79a and HLA-DR. CD38 was strongly expressed but CD20 was only weakly positive. Surface and cytoplasmic CD22 and immunoglobulin heavy chains and light chains were not detectable (Fig. 2). CD45 expression was strong and markers of immaturity including CD34 and terminal deoxynucleotidyl transferase (TdT) were not expressed. Lack of expression of multiple B-cell markers suggested a diagnosis of common acute lymphoblastic leukemia (cALL). However, the strong CD45 expression was unusual in acute leukaemia except for monoblasts. Neither was the absence of CD34 and TdT positivity supportive of ALL. Trephine biopsy revealed extensive infiltration by medium-sized to occasionally large abnormal lymphoid cells with round to irregular nuclei and multiple small nucleoli. A starry sky pattern was not evident (Fig 1b). Immunohistochemical staining on showed that the tumor cells were weakly positive for CD20. They strongly expressed BCL2 (Fig. 1c) but were negative for BCL6. Ki67 staining revealed less than 90\% positive cells. Strong BCL2 expression and a proliferation index less than 95\% preclude a diagnosis of BL. A germinal center-type DLBCL with loss of multiple B-lineage antigens was thus favored.

Conventional cytogenetic analysis revealed two related clones partially sharing 
multiple clonal abnormalities including amplification of 1(q21q32), t(2;18)(p12;q21), $\mathrm{t}(3 ; 22)(\mathrm{q} 27 ; \mathrm{q} 11.2),+7$, and $\mathrm{t}(8 ; 14)(\mathrm{q} 24 ; \mathrm{q} 32)$ with duplication of the derivative chromosome 14 and loss of its normal homologue. Such a complex karyotype was highly unusual in BL. The minute derivative chromosome 22 was morphologically identical to a Philadelphia chromosome but chromosomes 9 were normal (Fig. 1d). A three-way translocation $(3 ; 9 ; 22)(q 27 ; q 34 ; q 11.2)$ was suspected but subsequently excluded by a metaphase FISH analysis using BCR/ABL1 dual-color dual-fusion translocation probes (Abbott Laboratories, Abbott Park, IL) (Fig. 1e). A triple-hit B-cell lymphoma was suspected from the breakpoints observed at 3q27, 8q24 and 18q21, where BCL6, MYC and BCL2 reside, respectively. A metaphase FISH analysis using IGH/MYC dual-color dual-fusion translocation probes was performed (Abbott Laboratories, Abbott Park, IL), which confirmed IGH-MYC reciprocal translocation and duplication of derivative chromosome 14. In addition, an IGH signal was seen on the minute derivative chromosome 22 (Fig. 1f). Together with the $t(3 ; 22)$ observed in conventional cytogenetic analysis, findings suggested a 3-way translocation $\mathrm{t}(3 ; 8 ; 22)(\mathrm{q} 27 ; \mathrm{q} 24 ; \mathrm{q} 11.2) \mathrm{t}(8 ; 14)$. This involved a chromosome 3, a derivative chromosome 8 from $\mathrm{t}(8 ; 14)$ and a chromosome 22 . The second break in the $\mathrm{q}$ arm of the derivative chromosome 8 occurred distal to the first break, moving the telomeric portion of the translocated IGH gene to chromosome 22. Rearrangement of BCL6 in 
$\mathrm{t}(3 ; 8 ; 22)(\mathrm{q} 27 ; \mathrm{q} 24 ; \mathrm{q} 11.2) \mathrm{t}(8 ; 14)$ (Fig. 1g) and BCL2 in $\mathrm{t}(2 ; 18)(\mathrm{p} 12 ; \mathrm{q} 21)$ (data not shown) was confirmed by interphase FISH using respective probes (Abbott Laboratories, Abbott Park, IL).

The modest nuclear pleomorphism and the triple-hit complex karyotype were not classical of DLBCL. A diagnosis of B-cell lymphoma unclassifiable with features intermediate between DLBCL and BL was deemed most appropriate. The patient was given intensive combination chemotherapy Hyper-CVAD (cyclophosphamide, vincristine, doxorubicin, dexamethasone) but the response was poor. He died of refractory disease four months after presentation. 


\section{Discussion}

B-cell lymphoma unclassifiable with features intermediate between DLBCL and BL is a newly introduced category of mature B-cell lymphoma. Although specific genetic markers have not been identified in this group of lymphomas, they often show complex cytogenetics with frequent double-hit and occasional triple-hit changes. They are thought to strongly overlap with the molecular intermediate lymphomas defined by gene expression profiling [5]. The complex karyotype of our case provides many diagnostic clues but at the same time it is very challenging to dissect the various abnormalities. The exclusion of $B C R-A B L 1$ fusion in this case is of paramount importance because its presence indicates a diagnosis of Ph-positive ALL rather than a mature B-cell lymphoma. A chemotherapeutic regimen for ALL incorporating a tyrosine kinase inhibitor is then necessary [6]. Detection of $t(8 ; 14)(q 24 ; q 32)$, which suggests IGH-MYC translocation, does not itself exclude a diagnosis of B-cell ALL as rare cases of CD10 and TdT-positive precursor B-cell ALL with t(8;14)(q24;q32) have been reported [7-9].

Loss of immunoglobulin expression in high grade B-cell lymphoma indicated either the presence of non-functional somatic hypermutations in multiple immunoglobulin genes in lymphoma cells of germinal center origin or multiple chromosomal rearrangements disrupting these genes [1]. The absence of detectable 
immunoglobulin in tumor cells of this case is readily explained by the involvement of both $I G H$ genes in the same translocation with $M Y C$, leaving no functional $I G H$ for immunoglobulin expression. Translocation breakpoints at 2p12 and 22q11.2 also suggest involvement of $I G K$ and $I G L$, respectively. This is exceptional when compared to the usual double-hit lymphomas where typically one $I G H$ is involved in rearrangement with $M Y C$ and the other $I G H$ with BCL2, or less commonly BCL6. Lack of immunoglobulin expression alone in the absence of TdT or CD34 positivity should not be used to substantiate a diagnosis of precursor B-cell malignancy, especially when karyotyping shows IGH translocation [10].

The absence of detectable BCL6 expression in this case is rather unexpected [11]. Indeed, a demonstrable BCL6 rearrangement in the $t(3 ; 8 ; 22)(q 27 ; q 24 ; q 11.2)$ suggests IGL-BCL6 translocation at the breakpoints. The possibility of a missense mutation(s) in BCL6 leading to epitope change and immunohistochemical negativity cannot be excluded. This phenomenon has been observed in some BCL2-rearranged but “BCL2-negative” follicular lymphoma [12].

The disease in our patient nicely illustrates the importance of integrating morphological, immunophenotypic and genetic information to distinguish this B-cell lymphoma, unclassifiable, with features intermediate between DLBCL and BL from the classical BL and DLBCL. Guidelines for the differentiation have been provided by 
the WHO Classification of Tumours of Haematopoietic and Lymphoid Tissue [1]. 


\section{References}

1. Swerdlow SH, Campo E, Harris NL, Jaffe ES, Pileri SA, Stein H, eds. WHO Classification of Tumours of Haematopoietic and Lymphoid Tissue. Geneva: World Health Organization; 2008.

2. Perry AM, Crockett D, Dave BJ, Althof P, Winkler L, Smith LM, et al. B-cell lymphoma, unclassifiable, with features intermediate between diffuse large B-cell lymphoma and burkitt lymphoma: study of 39 cases. Br J Haematol. 2013 Apr 18. doi: 10.1111/bjh.12343. [Epub ahead of print]

3. Dave SS, Fu K, Wright GW, Lam LT, Kluin P, Boerma EJ, et al. Molecular diagnosis of Burkitt's lymphoma. N Engl J Med. 2006;354:2431-42.

4. Hummel M, Bentink S, Berger H, Klapper W, Wessendorf S, Barth TF, et al. A biologic definition of Burkitt's lymphoma from transcriptional and genomic profiling. N Engl J Med. 2006;354:2419-30.

5. Salaverria I, Siebert R. The gray zone between Burkitt's lymphoma and diffuse large B-cell lymphoma from a genetics perspective. J Clin Oncol. 2011;29:1835-43.

6. Liu-Dumlao T, Kantarjian H, Thomas DA, O'Brien S, Ravandi F. Philadelphia-positive acute lymphoblastic leukemia: current treatment options. Cur Oncol Rep. 2012;14:387-94.

7. Mufti GJ, Hamblin TJ, Oscier DG, Johnson S. Common ALL with pre-B-cell features showing $(8 ; 14)$ and $(14 ; 18)$ chromosome translocations. Blood. 1983;62:1142-6.

8. Pegoraro L, Palumbo A, Erikson J, Falda M, Giovanazzo B, Emanuel BS, et al. A $14 ; 18$ and an 8;14 chromosome translocation in a cell line derived from an acute B-cell leukemia. Proc Natl Acad Sci U S A. 1984;81:7166-70.

9. Navid F, Mosijczuk AD, Head DR, Borowitz MJ, Carroll AJ, Brandt JM, et al. Acute lymphoblastic leukemia with the (8;14)(q24;q32) translocation and FAB L3 morphology associated with a B-precursor immunophenotype: the Pediatric Oncology Group experience. Leukemia. 1999;13:135-41.

10. Komrokji R, Lancet J, Felgar R, Wang N, Bennett JM. Burkitt's leukemia with precursor B-cell immunophenotype and atypical morphology (atypical Burkitt's leukemia/lymphoma): case report and review of literature. Leuk Res. 2003;27:561-6.

11. de Jong D. Novel lymphoid neoplasms--the borderland between diffuse large B-cell lymphoma and Burkitt's lymphoma. Haematologica. 2009;94:894-6.

12. Masir N, Campbell LJ, Goff LK, Jones M, Marafioti T, Cordell J, et al. BCL2 protein expression in follicular lymphomas with $\mathrm{t}(14 ; 18)$ chromosomal translocations. Br J Haematol. 2009;144:716-25. 
Figure legends

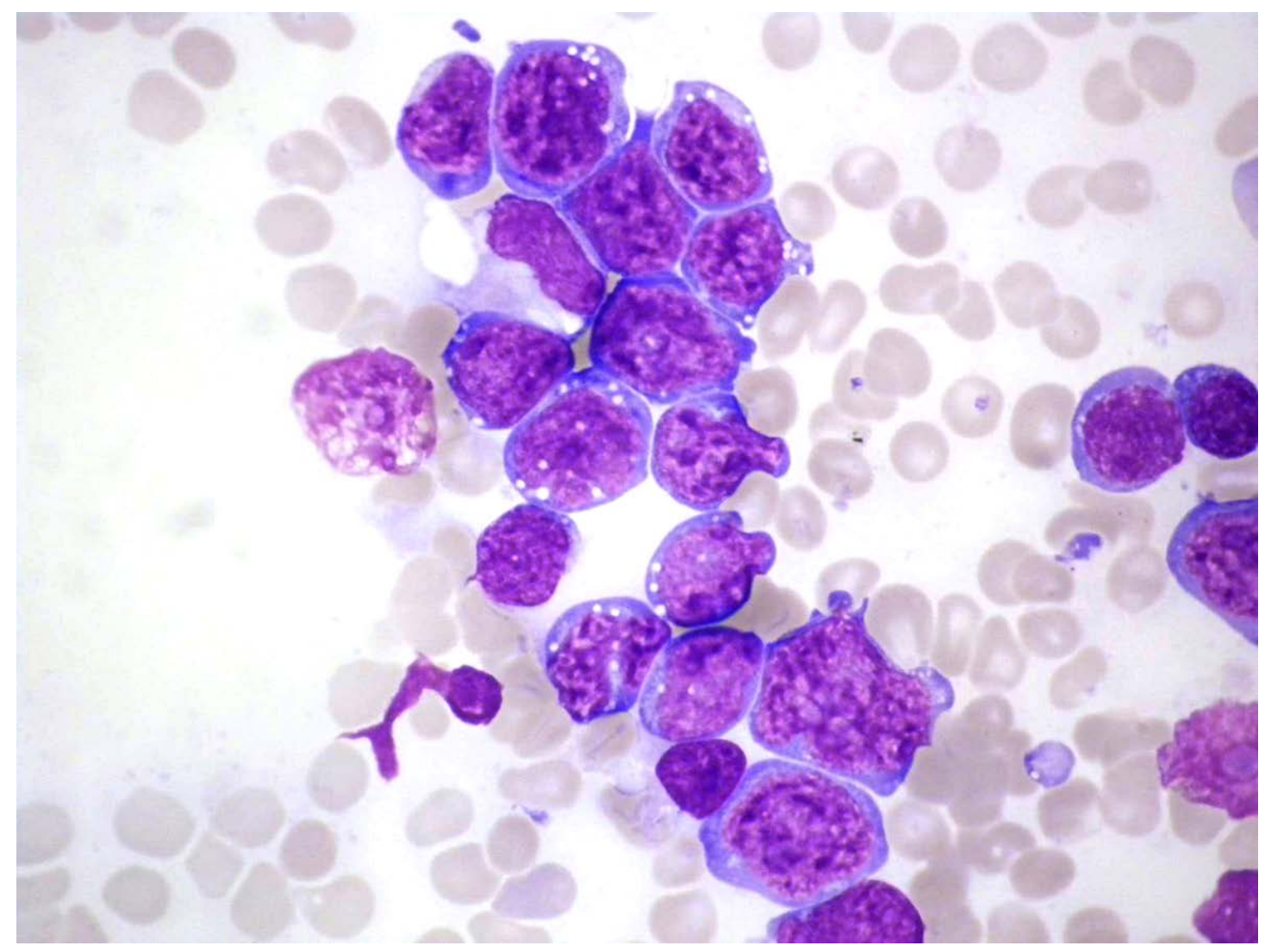

Figure 1. (a) Wright-Giemsa stained bone marrow aspirate smear shows a mixture of medium-sized and large abnormal lymphoid cells, 1000x original magnification. 


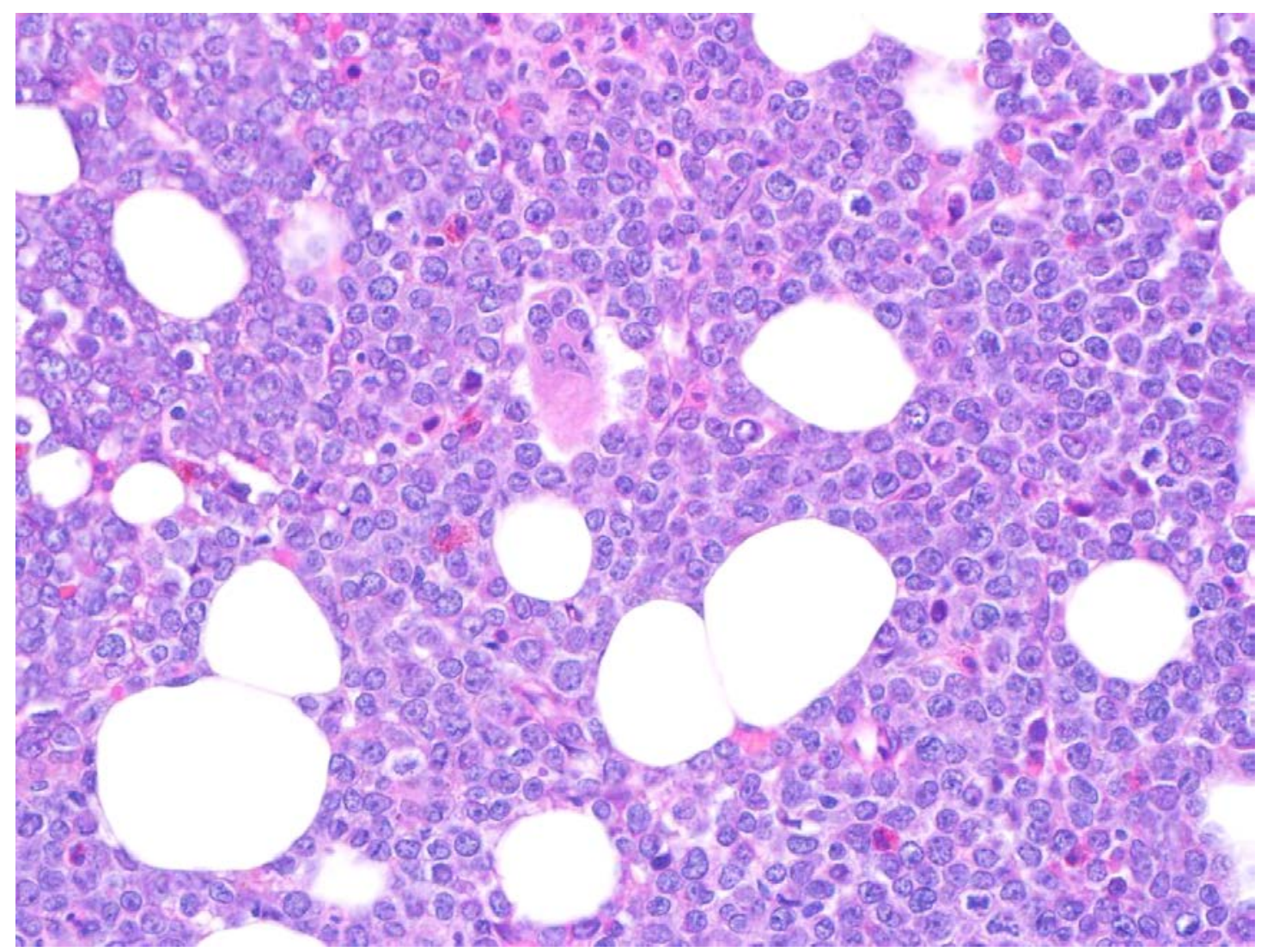

Figure 1. (b) Hematoxylin and eosin stained trephine biopsy shows a dense infiltration

of lymphoma cells that are mostly medium-sized, with no starry sky appearance, 400x original magnification. 


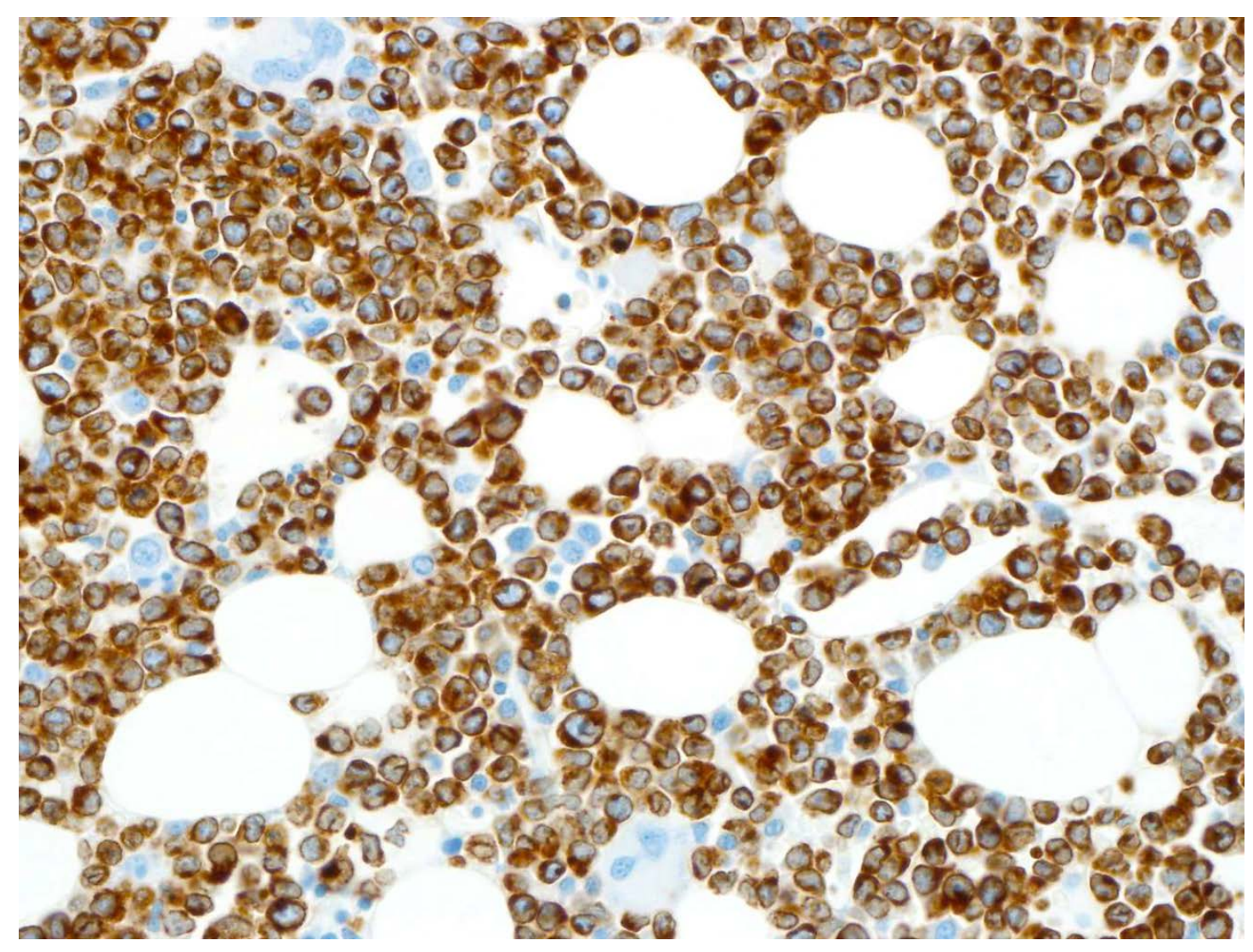

Figure 1. (c) Immunohistochemical staining for BCL2 shows strong positivity in tumor cells, 400x original magnification. 

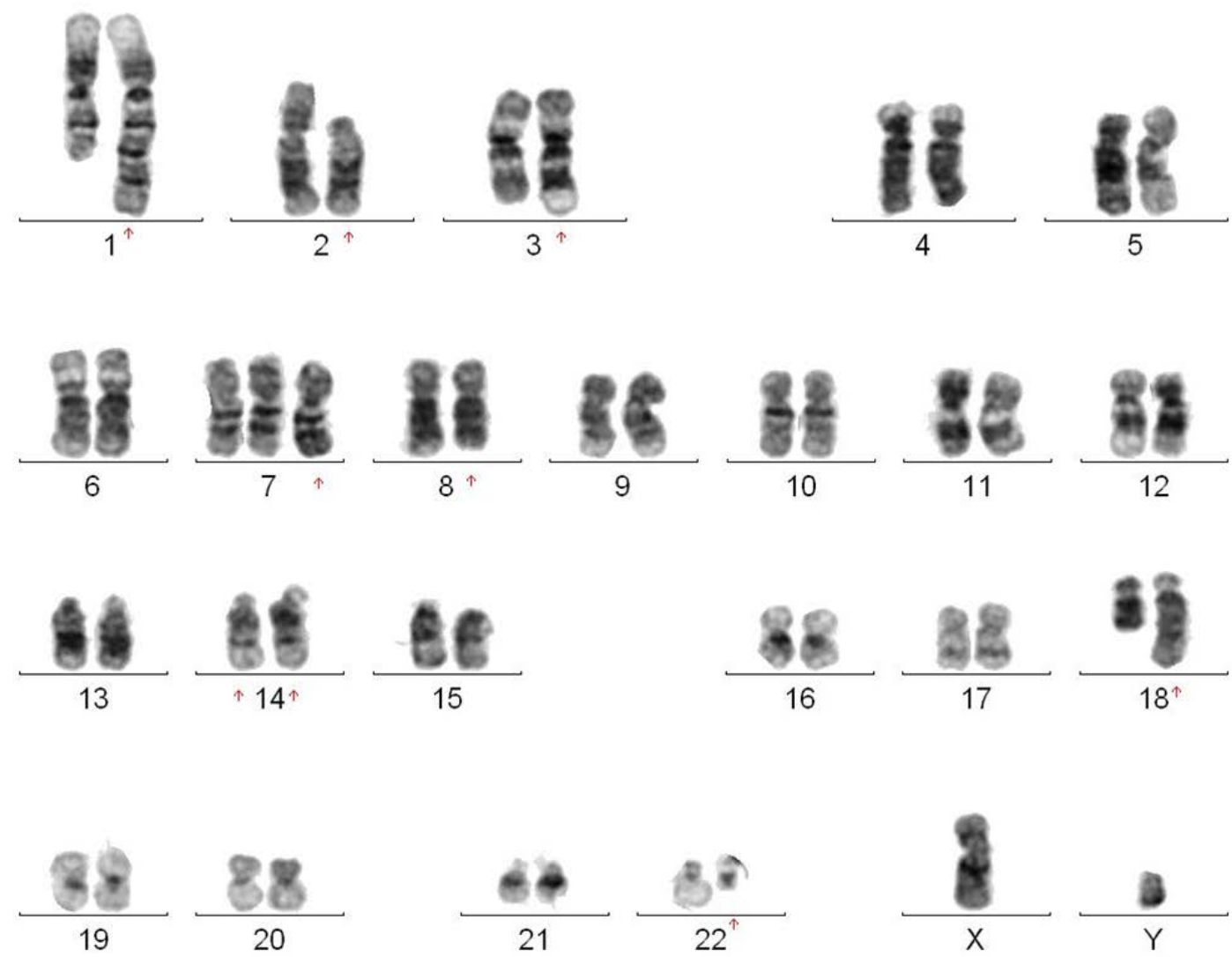

Figure 1. (d) A karyotype performed from marrow aspirate reveals multiple abnormalities, in particular t(2;18)(p12;q21), t(3;22)(q27;q11.2), and t(8;14)(q24;q32) with duplication of the derivative chromosome 14 and loss of its normal homologue. 

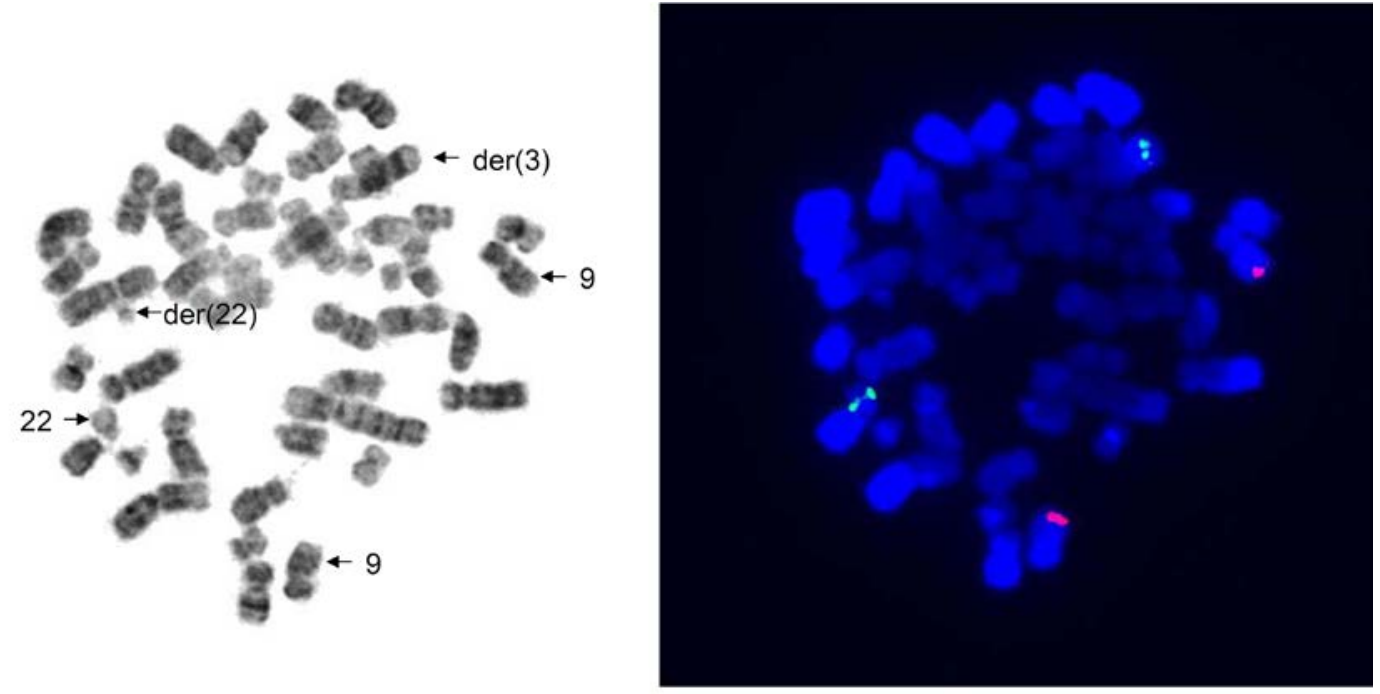

Figure 1. (e) Metaphase fluorescence in-situ hybridization (FISH) study using dual-color dual-fusion probes for $B C R$ and $A B L 1$ shows no gene fusion. One BCR gene (green signal) is translocated en bloc to chromosome 3 in the $\mathrm{t}(3 ; 22)(\mathrm{q} 27 ; \mathrm{q} 11.2)$.
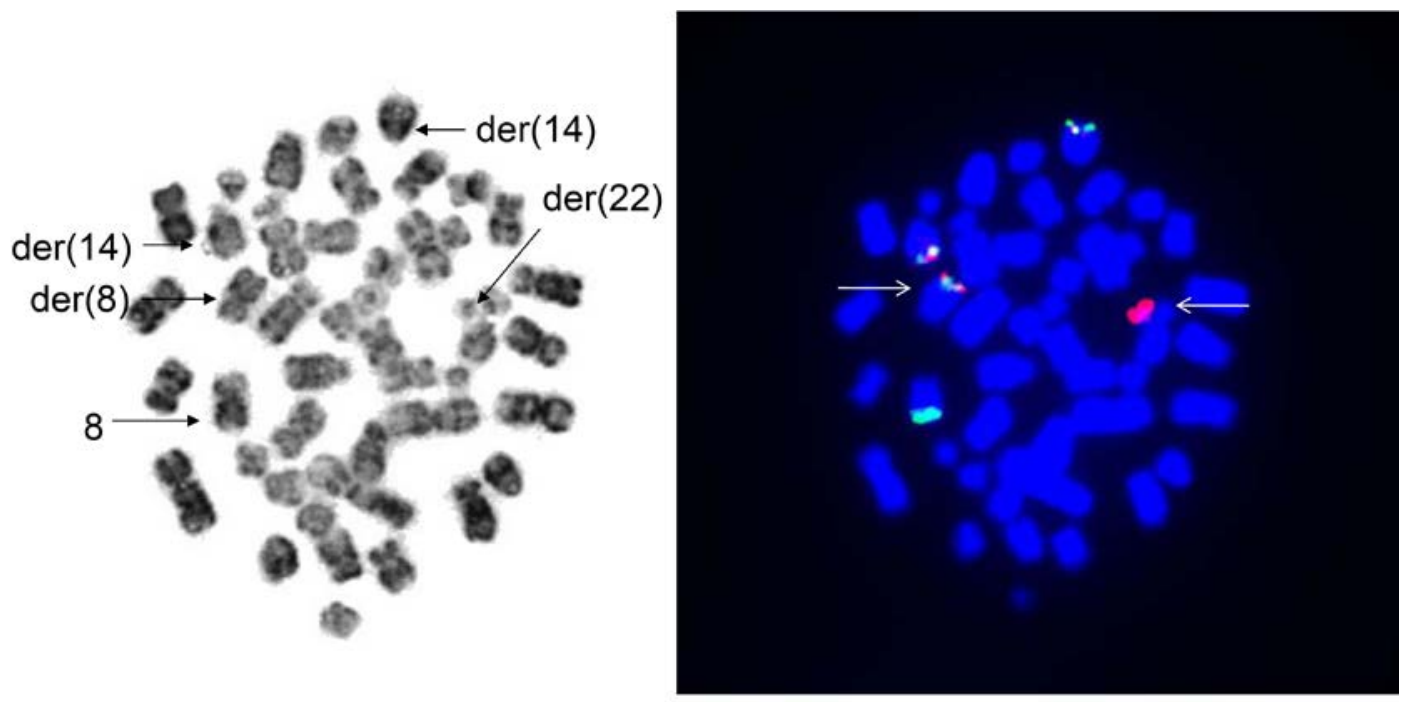

Figure 1. (f) Metaphase FISH study employing dual-color dual-fusion IGH (red) and MYC (green) probes confirms reciprocal IGH-MYC rearrangement on a derivative chromosome 8 and both derivative chromosome 14. An additional IGH signal is seen on the derivative chromosome 22 from the observed $\mathrm{t}(3 ; 22)(\mathrm{q} 27 ; \mathrm{q} 11.2)$. 


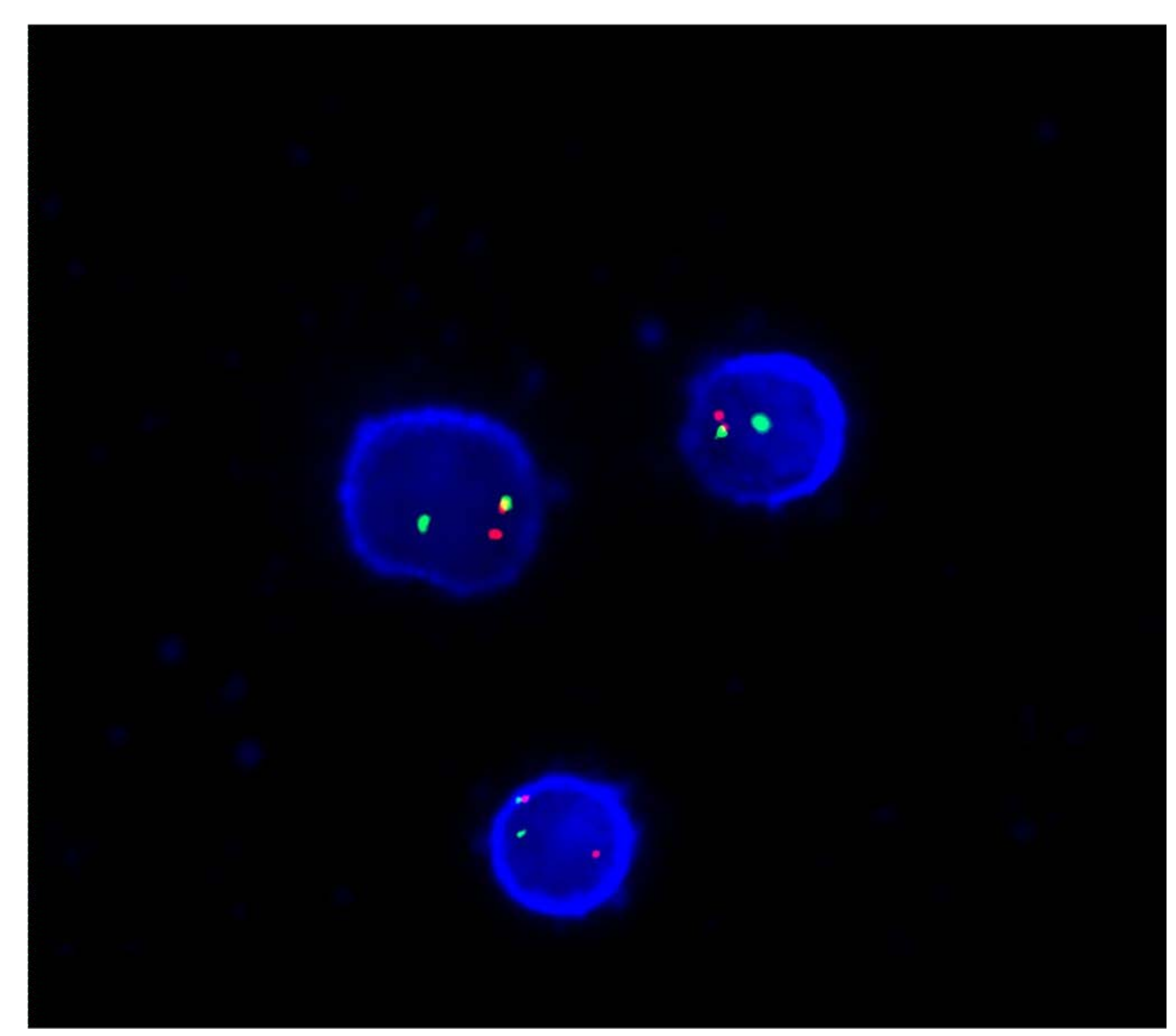

Figure 1. (g) Interphase FISH study using BCL6 dual-color break apart probes reveals splitting of one fusion signal signifying rearrangement of BCL6. 

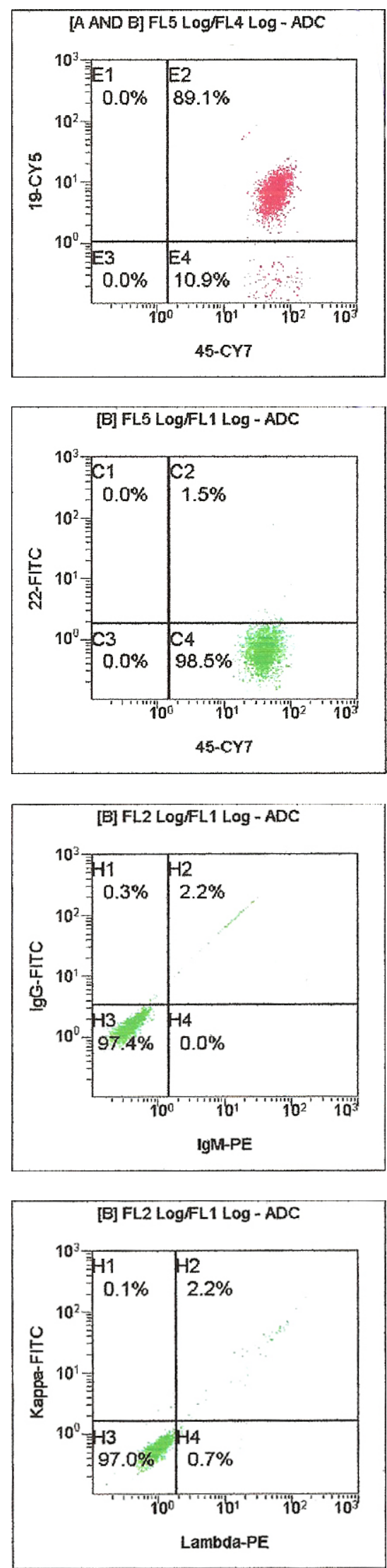
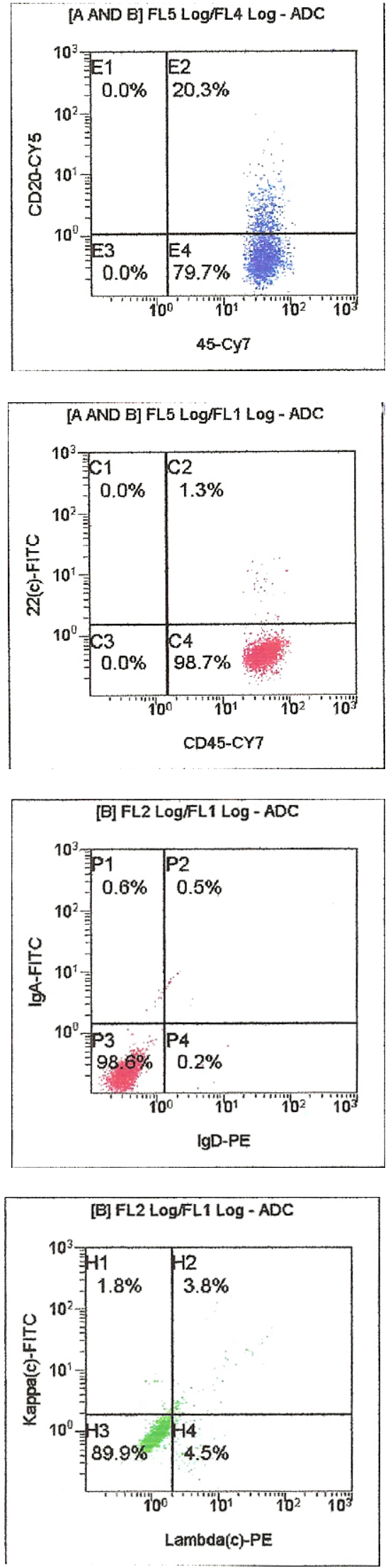

Figure 2. Scatter plots from flow cytometric analysis of marrow tumor cells show 
normal CD19, weak CD20 and no surface and cytoplasmic CD22 expression (upper panel, gated on CD45-positive and low side scatter lymphoid population). Surface immunoglobulin heavy chains and surface and cytoplasmic light chains are all negative (lower panel, gated on CD19-positive cells). 